\title{
Magnetic storm acceleration of radiation belt electrons observed by the Scintillating Fibre Detector (SFD) onboard EQUATOR-S
}

\author{
M. Cyamukungu ${ }^{1}$, C. Lippens ${ }^{2}$, L. Adams ${ }^{3}$, R. Nickson ${ }^{3}$, C. Boeder ${ }^{4}$, V. Pierrard ${ }^{2}$, E. Daly ${ }^{3}$, Gh. Grégoire ${ }^{1}$, \\ and J. Lemaire ${ }^{2}$ \\ ${ }^{1}$ UCL - Institut de Physique Nucléaire, Chemin du Cyclotron 2, B-1348 Louvain-la-Neuve, Belgium \\ ${ }^{2}$ BIRA/IASB - Avenue Circulaire 3, B-1180 Uccle, Belgium \\ ${ }^{3}$ ESA/ESTEC - P.O. Box 299, Keplerlaan 1, 2200 AG Noordwijk, The Netherlands \\ ${ }^{4}$ SENSYS - P.O. Box 411, 2201 AG Noordwijk, The Netherlands
}

Received: 1 February 1999 / Revised: 28 May 1999 / Accepted: 17 June 1999

\begin{abstract}
On the basis of the currents induced by electron fluxes in the Scintillating Fibre Detector (SFD) onboard the EQUATOR-S satellite launched on 2 December 1997, an in-situ acceleration of radiation belt electrons is found to possibly contribute to the increase of the flux of electrons with energies greater than $400 \mathrm{keV}$. The data acquired between 16 December 1997 and 30 April 1998 on the $500-67300 \mathrm{~km}, 4^{\circ}$ inclination EQUATOR-S orbit show that the increase of the energetic electron flux corresponds to the enhanced geomagnetic activity measured through the Dst index.
\end{abstract}

Key words. Magnetospheric physics (energetic particles, trapped; storms and substorms; instruments and techniques)

\section{Introduction}

The origin of energetic electrons observed in the outer radiation belt during magnetic storms remains a non fully understood issue since the 1960's. Phenomenological approaches have mainly resulted in a list of observables correlated with the geomagnetic storm intensity as measured for instance by the Dst index, but quantitative estimates of the electron spectrum variation due to each injection or acceleration mechanism are still needed (Mcllwain, 1996; Rostoker et al., 1998; Xinlin et al., 1998). Recently, the acceleration of electrons in the outer radiation belt has been attributed to Pc 5 band ULF waves excited by high speed solar wind flow associated with magnetic storms (Rostoker et al., 1998). The main features of this acceleration process are the local character of the observed flux enhancements of energetic electrons and the lack of

Correspondence to: M. Cyamukungu

e-mail: cyam@fynu.ucl.ac.be acceleration in the heart of the outer radiation belt $(L \approx 4)$. Finally, a comparison between the energy transferred to electrons and the energy content of ULF waves is suggested as a way to definitely attribute electron acceleration to this physical mechanism.

In this paper we show that the changes in the electron integral flux $J_{e}(E>0.4 \mathrm{MeV})$ observed by the Scintillating Fibre Detector (SFD) do not appear to correspond to injections of energetic electrons from outside the radiation belts and have affected the whole outer radiation belt regions for which $3<L<7$. The flux of high energy electrons and their extent in L-space appear to correspond to the intensity of the geomagnetic storm.

The SFD used to detect electron and proton fluxes is described in Sect. 2. Section 3 contains a discussion on the possible origin of the particles detected by the SFD.

\section{The instrument}

The Scintillating Fibre Detector (SFD) was designed and built by SENSYS under an ESA development contract and ESTEC assisted in the design of the shielding and in the calibration (Boeder et al. 1994). It contains two organic $1-\mathrm{mm}$ diameter (doped $\mathrm{C}_{8} \mathrm{H}_{8}$ ) scintillating sensors coupled to photodiodes by a PMMA optical fibre. In order to avoid solar light penetration into the satellite, a 0.3 -mm-thick aluminum foil constitutes the SFD front panel. The mechanical assembly of the SFD sensors is shown in Fig. 1. The shieldings in front and around each of these sensors determine different energy thresholds for the electrons and protons which the SFD was designed to detect. The aluminum shield around one of the organic scintillators is $0.2 \mathrm{~mm}$ thick, this channel is called the Naked Scintillating Fibre (NSF). The second organic scintillator is more heavily shielded with a 4-mm-thick aluminum tube and called the Shielded Scintillating Fibre (SSF). The threshold energies of electrons are $0.4 \mathrm{MeV}$ and $2 \mathrm{MeV}$ for the NSF and the SSF, respectively, while the corresponding values for protons are $8.6 \mathrm{MeV}$ and $30 \mathrm{MeV}$. 
The current from each photodiode is measured via a logarithmic amplifier allowing each channel to cover a wide range of currents (i.e. particle flux). The measured current is converted to particle flux using the experimentally determined response of the scintillating material to electrons or protons. The response of the NSF and SSF channels to electrons is $4.2 \times 10^{-7} \mathrm{~A} / \mathrm{W}$ in the $1 \mathrm{~cm}^{2}$ sensor section. It was deduced from a limited set of calibration data but proved to be underestimated by a large factor as indicated in Fig. 2 where the predicted NSF output current is compared to the observed one. The prediction was based on AE-8 and AP-8 models along with the response of the scintillating fibre channels to electrons and protons with energies above the given thresholds. The underestimated response to electrons given above leads to underestimated values of the predicted contribution of the electron flux to the NSF current. This can be seen by comparing the predicted NSF currents to observed values in the outer belt where the current signal is dominated by the electron contribution. The underestimation of the predicted NSF current when compared to the observed one is a general feature of all the data acquired during the EQUATOR-S mission. Therefore, the NSF current which is proportional to the integral electron flux $J_{e}(E>0.4 \mathrm{MeV})$ in the outer belt, can only be used to study the dynamics of the outer belt electrons.

The response of the NSF and SSF channels to protons was measured more accurately and is $2.2 \times 10^{-5} \mathrm{~A} / \mathrm{W}$ in the $1 \mathrm{~cm}^{2}$ sensor section. This allowed us to combine the
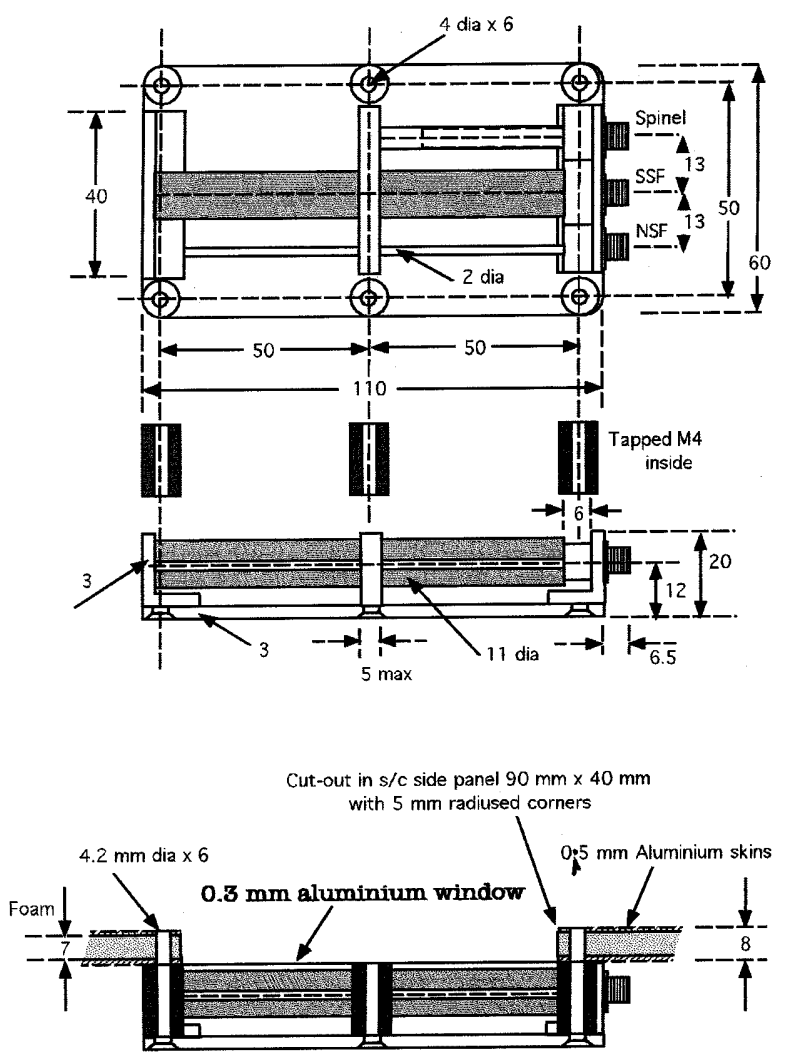

Fig. 1. Mechanical assembly of the Scintillating Fibre Detector built and calibrated by SENSYS/Noordwijk, under ESA contract No. 10988/94/NL/NB currents from the NSF and the SSF channels in order to determine the proton fluxes $J_{p}(E>8.6 \mathrm{MeV})$ along the EQUATOR-S orbit for $L<3$. A typical signal acquired
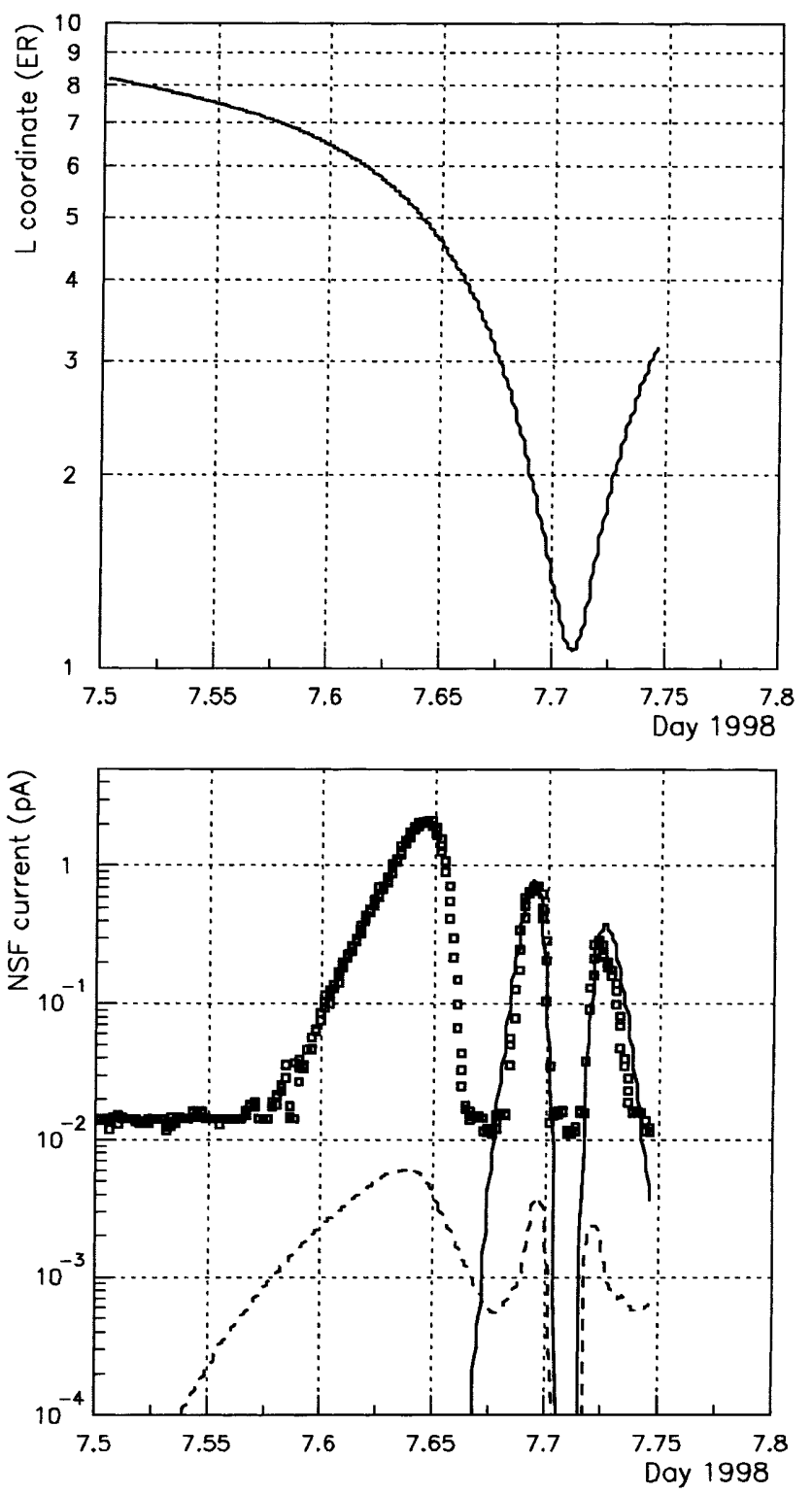

Fig. 2. Observed NSF output current (square symbols) versus time compared to the output current predicted using AP-8 and AE-8 models and the response of the scintillating fibres to protons and electrons. The upper panel gives the values of $L$ at which currents were measured. The predicted proton contribution (solid line) is either in good agreement with the observed currents where $L<3$ or slightly overestimated in the outer edge of the inner belt. The well known overestimation of the electron fluxes by the AE-8 model (Heck, 1992) should have resulted in an overestimated electron contribution to the NSF output current. But, due to the largely underestimated response of the scintillating fibres to electrons, the predicted electrons contribution (dashed line) is found to be a few orders of magnitude lower than the observed current in the outer belt where the electron contribution to the NSF current dominates. The predicted electron contribution to the NSF current at time 7.611 should be multiplied by a factor of about 30 in order to adjust its value to the observed one, but the same correction applied to the NSF current predicted for time 7.694 (in the inner belt) results in a relatively low electron contribution when compared to protons 


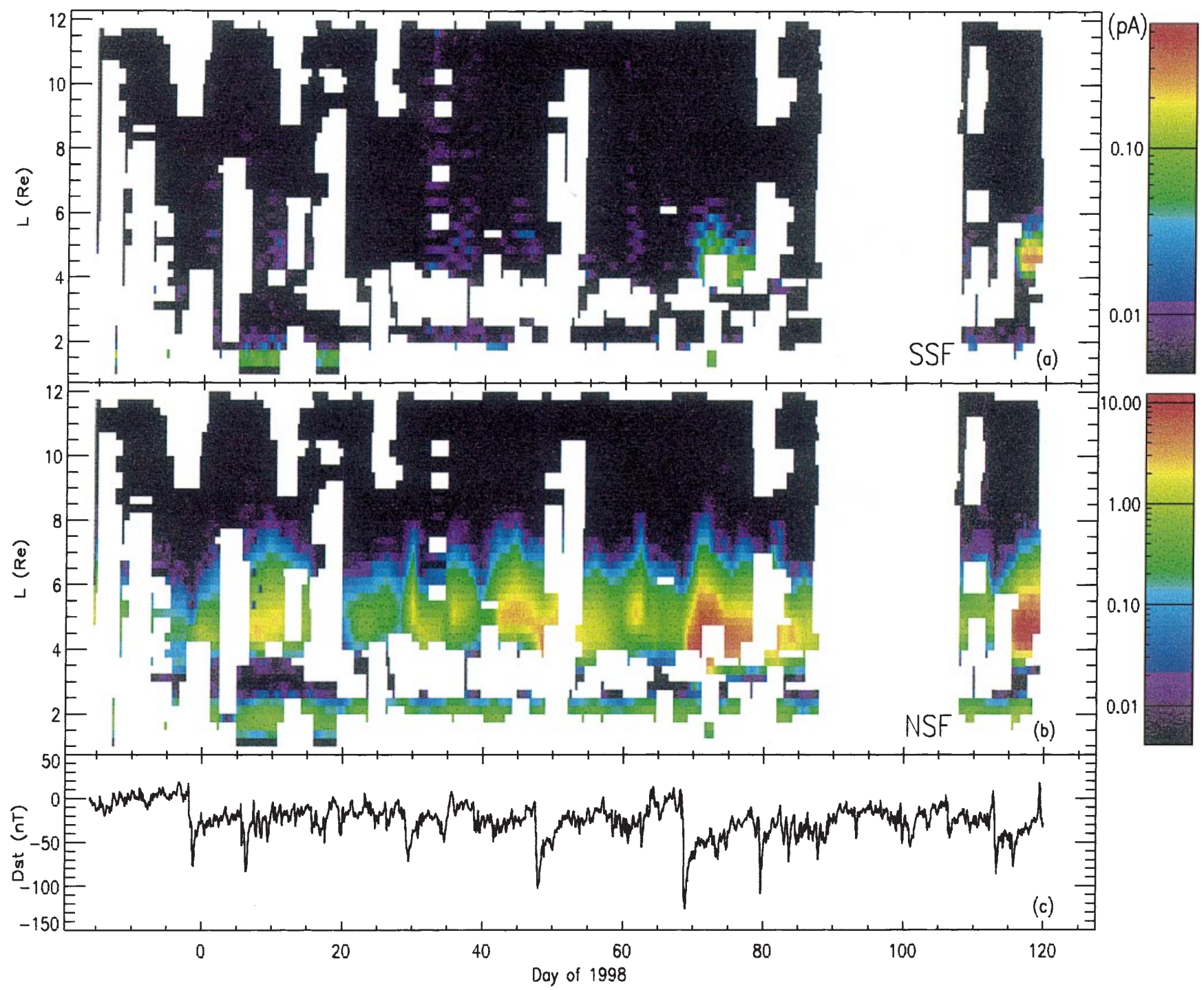

Fig. 3. Dynamical changes of the SSF (a) and the NSF (b) output current versus $L$ and time along the EQUATOR-S orbit. The height of the bins is 0.25 in $L$ and 0.25 days in time. A linear interpolation over a maximum of 10 time bins fills some of the data gaps between orbits.

in the inner belt region is also shown in Fig. 2. The amplitude of the predicted AP-8 proton contributions to the NSF current appears to be of the same order of magnitude as the observed one.

A correct electron calibration would have allowed us to extract the proton and electron fluxes from the observed SFD currents in case the latter had contained significant contributions from both particle types. However, all our analyses and predictions based on the AP-8 and AE-8 models (in particular, the agreement between the observed NSF current and the proton contribution in the inner edge) lead to the conclusion that protons are the main source of the observed NSF and SSF currents in the inner belt. Therefore, the currents measured by the NSF and SSF channels were converted to proton fluxes, which are only valid if the low contribution of electrons to the NSF current is confirmed by calculations based on a correct NSF response to electron fluxes.
The panel (c) corresponds to the Dst index from OMNIWeb Data Explorer (http://nssdc.gsfc.nasa.gov/omniweb/form/dx.html). Note the correlation between the SFD output current and the dips in the average Dst geomagnetic activity index

In the conversion of NSF and SSF currents to proton fluxes, the mean value of currents lower than $0.02 \mathrm{pA}$ outside the radiation belts was substracted from all the observed current values higher than $0.02 \mathrm{pA}$ to perform a baseline current correction. This baseline level amounts to $\approx 1.27 \times 10^{-2} \mathrm{pA}$ which correspond to a flux detection threshold of $\approx 500$ protons/ $\left(\mathrm{sec} \mathrm{cm}^{2} \mathrm{sr}\right)$.

A more detailed description of the SFD, its calibration and the proton flux results may be found in Boeder et al. (1994); Lemaire et al. (1997) and Cyamukungu et al. (1998).

\section{Results and discussion}

As stated above, our qualitative study of the dynamical changes of the electrons flux in the outer radiation belt (i.e at $L>3$ ) is based on raw output currents proportional to the flux of electrons above $0.4 \mathrm{MeV}$ (the proton 
contribution to the NSF and SSF currents is negligible in the outer belt region). The NSF signal was found to be intense on days of strong magnetic storms (Fig. 3b).

From Fig. 3a, it can be seen that the signal of the SSF channel rose significantly above the background mainly on days of magnetic storms, suggesting that only on these days electrons of $E>2 \mathrm{MeV}$ were injected or accelerated in the outer radiation belt. The SFD data displayed in Fig. 3(a and b), between day 107 (17 April) and day 120 (30 April, i.e. immediately after the wide data gap) could be most valuable in evaluating the radiation environment just before the latchup which resulted in the early termination of the EQUATOR-S mission; therefore, these SFD data sets offer the opportunity to help clarify the origin of this unfortunate abrupt end of mission. No evidence of slowparticle, diffusive-like transport from the outer belt to the inner belt has been found (see Fig. $3 a$ and $3 b$ ). Figures 2 and 3 ( $a$ and $b$ ) show that no particle bursts were detected outside the radiation belts. This may be due to either of the following causes: (i) solar wind particle injections occurred at high altitude at the time when the EQUATOR-S satellite was at low altitude; (ii) the flux of particles coming from outside the belts was of so low a level that the SFD did not detect it; (iii) there was no injection of high energy charged particles from outside the belts on days with intense geomagnetic storms.

The correlation between changes in the flux of outer belt electrons and solar wind parameters has been reported by Blake et al. (1997), Selesnick and Blake (1998) and Xinlin et al. (1998) and the short duration of the revealed injection process (several hours or less) along with the orbital period of EQUATOR-S (22.3 h) may explain that the SFD was never at the right position to detect particle bursts from outside the belts. This would be in favour of hypothesis (i) although it must be recalled that the EQUATOR-S satellite spent more than $90 \%$ of its time at positions for which $L>3$ (see the upper panel of Fig. 2).

On the other hand, if hypothesis (ii) applies then an upper limit on the flux of injected electrons could be determined if a correct calibration of the SFD for electrons were available. The characteristics of this flux would be found to be different from those of the flux detected by the SFD in the radiation belts. This would indicate that the observed high energy electrons are not necessarily those of lower flux level injected from outside the belts.

Finally, the most probable hypothesis one can draw on the basis of the SFD results is hypothesis (iii), which is compatible with electron acceleration by ULF waves as suggested by Rostoker et al. (1998). As for the case (ii), admitting hypothesis (iii) leads to the conclusion that most of the high energy electrons observed in the belts are accelerated internally and do not result from direct transport from outside the belts.

The observations made using the SFD favour a global approach to the study of the origin of high energy particles in the radiation belts. The dynamical properties of the radiation belts may be better studied by making a balance of the total incoming energy (irrespective of the radiation type) rather than only monitoring the particle injections into the belts. The adiabatic processes involved with in-situ acceleration of outer and inner belt electrons may then be characterized by the change induced in the whole energy and angular spectra of trapped particles.

Acknowledgements. The evaluation of the SFD and its data analysis were funded under ESTEC Contract No 11711/95/NL/ JG(SC). The authors thank D. Heynderickx (BIRA) and M. Kruglanski for their assistance in using UNIRAD and UNILIB and for discussions of the SFD results. We are very grateful to P. Nieminen for his advices and critical remarks during the analysis of these data.

Topical Editor K.-H. Glassmeier thanks B. Blake and C. Dyer for their help in evaluating this paper.

\section{References}

Blake, J. B., D. N. Baker, N. Turner, K. W. Ogilvie, and R. P. Lepping, Correlation of changes in the outer-zone relativisticelectron population with upstream solar wind and magnetic field measurements, Geophys. Res. Lett., 24, 927-929, 1997.

Boeder, C., L. Adams, and R. Nickson, Scintillating fibre detector system for spacecraft dosimetry, Proceedings of RADECS '93 IEEE 1994.

Cyamukungu, M., Gh. Grégoire, J. F. Lemaire, C. Lippens, and V. Pierrard, The scintillating fibre detector (SFD), technical note B (www.fynu.ucl.ac.be/projects/he/equator/gindex.html) - Louvain-la-Neuve, 1998.

Heck, F., Observation of radiation environment with CRRES data, ESA/ESTEC Report - March 1992.

Lemaire, J. F., C. Lippens, V. Pierrard, M. Cyamukungu, and Gh. Grégoire, The scintillating fibre detector (SFD), technical note A (www.fynu.ucl.ac.be/projects/he/equator/gindex.html) - Louvain-la-Neuve, 1997.

McIlwain, C. E., Process acting upon outer zone electrons in Radiation Belts: Models and Standards - Geophysical Monograph 97; American Geophysical Union. eds J.F. Lemaire, D. Heynderickx and D. N. Baker, pp 15-26, 1996.

Rostoker, G., S. Skone, and D. N. Baker, On the origin of relativistic electrons in the magnetosphere associated with some geomagnetic storms, Geophys. Res. Lett., 25, 3701-3704, 1998.

Selesnick, R. S., J. B. Blake, Radiation belt electron observations following the January 1997 magnetic cloud event, Geophys. Res. Lett., 25, 2553-2556, 1998.

Xinlin, Li., D. N. Baker, M. Temerin, T. Cayton, G. D. Reeves, T. Araki, H. Singer, D. Larson, R. P. Lin, and S. G. Kanekal, Energetic electron injections into the inner magnetosphere during the Jan. 10-11, 1997 magnetic storm, Geophys. Res. Lett., 25, 2561-2564, 1998. 